\title{
Acoustic Radiation from Compressor Shells
}

\author{
Y.V. Siva Prasad, Chandramouli Padmanabhan and N. Ganesan \\ Machine Dynamics Laboratory, Department of Applied Mechanics, \\ Indian Institute of Technology Madras, Chennai 600036, India
}

(Received 10 November 2003; accepted 4 February 2004)

\begin{abstract}
Structure-borne refrigeration compressor noise reduction has been a major concern for the industry. Structural modifications for noise reduction have often been carried out on the basis of a vibration or modal analysis of the compressor shell alone. Since there are two dense fluids inside a compressor shell, refrigerant and lubricating oil, significant dynamic interaction between the shell and the fluids is likely to occur. The major focus of this paper is to examine the transmission loss of such shells, considering the fluid-structure coupling. A semi-analytical finite element method is used to find the vibration response of the shell-fluid coupled system when excited by acoustic pulsations in the shell cavity caused by the reciprocating mechanism of the compressor. To calculate the acoustic and structural response, a special modal superposition technique based on adjoint eigenvectors is used since the finite element formulation leads to unsymmetric matrices. Once the structural vibration response has been computed, an axisymmetric boundary integral technique is used to predict acoustic pressures on the outside surface of the compressor shell. The transmission loss is then computed by comparing the acoustic pressures inside the cavity with the pressures on the outside surface. It is demonstrated that transmission loss is high for the first few modes of the shell and decreases with increase in the circumferential mode number. For a given circumferential mode the transmission loss is shown to increase with increase in axial mode number.
\end{abstract}

\section{INTRODUCTION}

Noise radiation from hermetic refrigeration compressors has been the subject of investigation for almost two decades now. Gas pulsation has been identified as one of the major sources responsible for the noise generation. Due to the finite volumes associated with the suction and discharge plenums and the reciprocating motion of the piston-cylinder mechanism, pressure pulsations occur in the acoustic cavity between the shell and the rigid block housing the motor and pistoncylinder mechanism. This shell cavity is usually composed of two fluids - refrigerant and lubrication oil. The high density associated with these fluids, relative to air, can cause fluid loading on the structure. This in turn influences the dynamic behaviour of the shell and changes the noise radiation from the shell structure.

Pritchard (1966) analysed the transmission of sound through a finite, closed cylindrical shell. From his analysis, two important frequencies for noise radiation were established, a) the ring frequency, where the wavelength of a longitudinal wave in the material is equal to the circumference and b) the coincidence frequency, where the trace wavelength of the incident wave is equal to the bending wavelength in the shell wall. Petyt and Lim (1978) have predicted the noise inside a mechanically excited, fluid-filled flexible cylinder. The coupled equations of motion for the cylinder and acoustic field were solved using modal analysis techniques.

Kim and Soedel (1989) have analysed the acoustic characteristics of multi-connected continuous acoustic elements. A general solution procedure has been developed for two continuous cavities that are multi-connected by onedimensional continuous pipes. Analysis was carried out using four pole concepts to obtain acoustic responses in terms of pulsating pressure and volume flow. As an application example, they considered the refrigeration compressor for under- standing the pressure pulsations inside the shell, with the acoustic input being the suction part of the compressor and the output being the evaporator pipe. They discussed the relative locations for input and output ports, pressure distributions in the cavity at resonance condition, and phase cancellations when two input ports are used. Kung and Singh (1985) developed a finite element technique to find the natural frequencies and modes of undamped three-dimensional acoustic cavities representing typical refrigeration compressors. The results of an annular like acoustic cavity were compared with experiments and good agreement established. Zhou and Kim (1996) have predicted the noise radiation of hermetic compressors by a combination of FE and boundary element (BE) methods. A numerical procedure for combining the computer simulation program for gas pulsations in the compressor and FE/BE methods has been developed by them to calculate the acoustic inputs to the cavity of the compressor. Pan and Jones (1999) have made an analysis on predicting the noise radiation from a spherically shaped reciprocating compressor. They compared the results obtained by analytical methods with those of experiments but missed out the rigid body mode in their analysis.

While the literature review does indicate that several studies on prediction of noise from shell systems are available, few are concerned with noise radiation in the presence of multiple fluids in the acoustic cavity. The influence of the fluid loading on noise has not been looked into earlier, and the primary goal of this paper is to examine this issue in detail. For this a typical hermetically sealed refrigeration compressor that contains two separate fluids: the refrigerant fluid and the lubricating oil, is considered. Response studies are done using a coupled unsymmetric finite element (FE) formulation. The formulation has been validated with results available from the literature as well as with experiments on a typical shell-fluid system. Analysis has been carried out to find out the noise radiation from the proposed compressor 